\title{
ON THE CONVERSE OF THE TRANSITIVITY OF MODULARITY
}

\author{
Y. K. WONG
}

E. H. Moore's theorem on the transitivity of modularity is as follows: Consider the basis ${ }^{1} \mathfrak{A}, \mathfrak{B}, \epsilon$; if a positive hermitian matrix $\epsilon_{0}$ is modular as to $\epsilon \epsilon$, then every vector which is modular as to $\epsilon_{0}$ is modular ${ }^{2}$ as to $\epsilon$ (that is, $\mathfrak{M}_{\epsilon_{0}} \subset \mathfrak{M}_{\epsilon}$ ).

In his doctoral thesis, the author establishes the converse of the preceding theorem as a consequence of the Hellinger-Toeplitz theorem. ${ }^{3}$ In this note, we give a new proof for the converse of the transitivity of modularity, and then deduce the generalized HellingerToeplitz theorem as a corollary. The converse of the transitivity of modularity is, therefore, equivalent to the Hellinger-Toeplitz theorem. We also establish the converse of the transitivity of modularity for matrices, and a theorem on the transitivity of accordance and finiteness.

Theorem I. Consider the basis $\mathfrak{A}, \mathfrak{B}, \epsilon$; and let $\epsilon_{0}$ be a positive hermitian matrix. Then the following assertions are equivalent:

(1) every vector $\mu_{0}$ modular as to $\epsilon_{0}$ is modular as to $\epsilon$;

(2) $\epsilon_{0}$ is modular as to $\epsilon \epsilon_{0}$;

(3) $\epsilon_{0}$ is modular as to $\epsilon \epsilon$.

If one of the preceding conditions is satisfied, the modulus of $\epsilon_{0}$ as to $\epsilon \epsilon$ is equal to the norm of $\epsilon_{0}$ as to $\epsilon \epsilon_{0}$.

In the course of demonstration, we let $\mathfrak{M}_{0}$ denote the space of vectors $\mu_{0}$ modular as to $\epsilon_{0} ; J_{0}$, the integration process based on $\epsilon_{0}$; and $M_{0}$, the modulus as to $\epsilon_{0}$. Similar interpretations are given to the symbols $\mathfrak{M}, J, M$, for the base matrix $\epsilon$. A vector which is finite as to $\epsilon$ is denoted by $\beta$.

If every $\mu_{0}$ is modular as to $\epsilon$, the matrix $\epsilon_{0}$ is of type $\mathfrak{M}_{0} \overline{\mathfrak{M}}$. Then $J \epsilon_{0} \beta$ is in $\mathfrak{M}_{0}$ for every $\beta$, and $J_{0}\left(J \bar{\beta} \epsilon_{0}\right) \mu_{0}=J \bar{\beta} J_{0} \epsilon_{0} \mu_{0}=J \bar{\beta} \mu_{0}$ for every pair $\beta, \mu_{0}$. Consequently, for every $\beta, M_{0} J \epsilon_{0} \beta$ is equal to the least upper bound of $\left|J \bar{\beta} \mu_{0}\right|$ for all $\mu_{0}$ such that $M_{0} \mu_{0} \leqq 1$, by part (2) of Theorem (41.9) in G.A. Similarly, for every $\mu_{0}$, which is modular as to $\epsilon$ by hypothesis, $M \mu_{0}$ is equal to the least upper bound of $\left|J \bar{\beta} \mu_{0}\right|$

${ }^{1}$ E. H. Moore, General Analysis (G.A. for abbreviation), Part I, p. 4, and Part II, p. 84 .

2 Theorem (46.4), part (1) in G.A., II, p. 137.

${ }^{3}$ Spaces associated with non-modular matrices with applications to reciprocals, Chicago thesis, 1931, pp. 3-9. The same proof is given in G.A., II, p. 193. 
for all $\beta$ such that $M \beta \leqq 1$. If the class $\mathfrak{R}$ is identified as the class of vectors $\bar{\beta}$ such that $M \beta \leqq 1$, and $F_{l}$, on $\mathfrak{M}_{0}$ to $\mathfrak{N}$, is defined to be $\left(\left|J \bar{\beta} \mu_{0}\right| \mid \mu_{0}\right)$ for every $l=\bar{\beta}$, then by Theorem (53.55) in G.A., ${ }^{4}$ the upper bound of $M_{0} J \epsilon_{0} \beta$, for all $\beta$ finite as to $\epsilon$ such that $M \beta \leqq 1$, is finite. By Theorem (46.85) in G.A., $\epsilon_{0}$ is modular as to $\epsilon_{0} \epsilon$. Since $\epsilon_{0}$ is hermitian, $\epsilon_{0}$ is also modular ${ }^{5}$ as to $\epsilon \epsilon_{0}$.

When condition (2) is true, then condition ( 3 ) is secured by a simple application ${ }^{6}$ of the composition of modularity ${ }^{7}$ to $\epsilon_{0}=J_{0} \epsilon_{0} \epsilon_{0}$. That the last condition implies the first is proved in Theorem (46.4) of Moore's G.A.

From $\epsilon_{0}=J_{0} \epsilon_{0} \epsilon_{0}$ and part (2) of Theorem (46.9) in G.A., we have $N_{\epsilon_{0} \epsilon} \epsilon_{0}=M_{\epsilon \epsilon} J_{0} \epsilon_{0} \epsilon_{0}=M_{\epsilon \epsilon} \epsilon_{0}$. This completes the proof.

The hypothesis of the preceding theorem is assumed for the following corollary:

Corollary. Let $\mathfrak{M}_{0 *}$ consist of all $\mu_{0}$ whose moduli as to $\epsilon_{0}$ are bounded by a fixed constant. If $\mathfrak{M}_{0 *}$ is a subset of $\mathfrak{M}$, then the moduli as to $\epsilon$ of all vectors in $\mathfrak{M}_{0 *}$ are also bounded.

We may assume, without losing generality, that the moduli of all vectors in $\mathfrak{M}_{0 *}$ are at most unity. Since the spaces $\mathfrak{M}_{0}$ and $\mathfrak{M}$ are linear, the condition that every $\mu_{0}$ for which $M_{0} \mu_{0} \leqq 1$ is modular as to $\epsilon$ is equivalent to condition (1) in the preceding theorem. Consequently $\epsilon_{0}$ is modular as to $\epsilon \epsilon_{0}$. The equation $\mu_{0}=J_{0} \epsilon_{0} \mu_{0}$ gives, by Theorem (46.7) in G.A., that $M \mu_{0} \leqq M_{\epsilon \epsilon_{0}} \epsilon_{0}$ whenever $M_{0} \mu_{0} \leqq 1$.

Theorem II. Consider the basis $\mathfrak{A}, \mathfrak{P}^{1}, \mathfrak{P}^{2}, \epsilon^{1}, \epsilon^{2}$; and let $\epsilon_{0}^{1}, \epsilon_{0}^{2}$ be two positive hermitian matrices. Then the following assertions are equivalent:

(1) every matrix $\kappa^{12}$ modular as to $\epsilon_{0}^{1} \epsilon_{0}^{2}$ is of type $\mathfrak{M}^{1} \overline{\mathfrak{M}}^{2}$;

(2) $\epsilon_{0}^{1}$ is modular as to $\epsilon^{1} \epsilon^{1}$, and $\epsilon_{0}^{2}$ is modular as to $\epsilon^{\mathbf{2}} \epsilon^{2}$;

(3) every matrix $\kappa^{12}$ modular as to $\epsilon_{0}^{1} \epsilon_{0}^{2}$ is modular as to $\epsilon^{1} \epsilon^{2}$.

For the demonstration of the theorem, we shall show that $(1) \rightarrow(2) \rightarrow(3) \rightarrow(1)$. The second implication is proved in part (2) of Theorem (46.4) in G.A. The last implication follows from the fact that every matrix $\kappa^{12}$ modular as to $\epsilon^{1} \epsilon^{2}$ is of type $\mathfrak{M}^{1} \overline{\mathfrak{M}}^{2}$. To show

${ }^{4}$ See also Hildebrandt, On uniform limitedness of sets of functional operations, this Bulletin, vol. 29 (1923), pp. 309-315; Fréchet, Sur les fonctionelles bilinéaires, Transactions of this Society, vol. 16 (1915), pp. 217-218.

5 By a similar reasoning, we may, of course, deduce the Hellinger-Toeplitz theorem as a consequence of Theorem (53.55).

6ee the author's thesis, loc. cit., p. 8, or Moore, G.A., II, p. 193.

7 Moore, G.A., II, p. 144. 
the first implication, consider any $\mu_{0}^{1}, \mu_{0}^{2} \neq 0^{2}$ which are modular as to $\epsilon_{0}^{1}, \epsilon_{0}^{2}$ respectively, Theorem (47.2) in G.A. shows that $\mu_{0}^{1} \bar{\mu}_{0}^{2}$ is modular as to $\epsilon^{1} \epsilon^{2}$, and hence by hypothesis, $\mu_{0}^{1} \mu_{0}^{2}$ is of type $\mathfrak{M}^{1} \overline{\mathfrak{M}}^{2}$. Since $\mu^{2} \neq 0^{2}$, let $a \equiv \mu^{2}\left(p^{2}\right) \neq 0$. Then $\mu_{0}^{1} \cdot a$, and hence $\mu_{0}^{1}$, is modular as to $\epsilon^{1}$. This proves that every $\mu_{0}^{1}$ modular as to $\epsilon_{0}^{1}$ is modular as to $\epsilon^{1}$. By Theorem I, $\epsilon_{0}^{1}$ is modular as to $\epsilon^{1} \epsilon^{1}$. Similarly, we prove that $\epsilon_{0}^{2}$ is modular as to $\epsilon^{2} \epsilon^{2}$. The proof was suggested by Dr. Coral.

Theorem III. (Generalized Hellinger-Toeplitz theorem.) Consider the basis $\mathfrak{A}, \mathfrak{P}^{1}, \mathfrak{B}^{2}, \epsilon^{1}, \epsilon^{2}$. A matrix $\kappa^{12}$ is modular as to $\epsilon^{1} \epsilon^{2}$ if and only if $\kappa^{12}$ is by rows of $\overline{\mathfrak{M}}^{2}$ and $J^{2} \kappa^{12} \mu^{2}$ is modular as to $\epsilon^{1}$ for every $\mu^{2}$.

To prove the theorem, we make use of the fact that $\kappa^{12}$ is modular as to $\epsilon^{1} \epsilon^{2}$ if and only if the following condition holds:

(M) $\kappa^{12}$ is by rowes of $\overline{\mathfrak{M}}^{2}$, and $J^{2} \kappa^{12} \kappa^{* 21}$ is modular as to $\epsilon^{1} \epsilon^{1}$.

This is Theorem (46.9) in G.A., with the omission of the redundant condition that $\kappa^{12}$ is by columns accordant as to $\epsilon^{1}$. (For when $\kappa^{12}$ satisfies the conditions (M), $\kappa^{12}$ is by columns $A^{1}$. To prove this, we note that $J^{2} \kappa^{12} \kappa^{* 21}$ is $A^{11}$ by Theorem (46.65) in G.A. Consequently, when $S_{\sigma}^{1} \epsilon^{1} \alpha^{1}=0^{1}$, then $J^{2}\left(S_{\sigma}^{1} \bar{\alpha}^{1} \kappa^{12}, S_{\sigma}^{1} \kappa^{* 21} \alpha^{1}\right)=S_{\sigma}^{1} S_{\sigma}^{1} \bar{\alpha}^{1} J^{2} \kappa^{12} \kappa^{* 21} \alpha^{1}=0$, which implies that $S_{\sigma}^{1} \bar{\alpha}^{1} \kappa^{12}=0^{2}$, since $J^{2}$ is proper. Thus $\kappa^{12}$ is by columns $A^{1}$.) Consequently, it suffices to prove the following statement: When $\kappa^{12}$ is by rows of $\overline{\mathfrak{M}}^{2}$, the matrix $J^{2} \kappa^{12} \kappa^{* 21}$ is modular as to $\epsilon^{1} \epsilon^{1}$ if and only if $J^{2} \kappa^{21} \mu^{2}$ is modular as to $\epsilon^{1}$ for every $\mu^{2}$.

Using the notation introduced by E. H. Moore in his study of generalized Fourier theory, we denote the positive hermitian matrix $J^{2} \kappa^{21} \kappa^{* 21}$ by $\epsilon_{\kappa^{*}}^{1}$. It was shown by Moore that the space of vectors modular as to $\epsilon_{\kappa^{*}}^{1}$ is equal ${ }^{8}$ to the space of vectors $J^{2} \kappa^{12} \mu^{2}$ for all $\mu^{2}$ in $\mathfrak{M}^{2}$. When $\kappa^{12}$ is assumed to be by rows of $\overline{\mathfrak{M}}^{2}$, the assertion that $J^{2} \kappa^{12} \mu^{2}$ is modular as to $\epsilon^{1}$ for every $\mu^{2}$ is equivalent to the assertion that every vector modular as to $\epsilon_{\kappa^{*}}^{1}$ is modular as to $\epsilon^{1}$. By Theorem I, the latter assertion is valid if and only if $\epsilon_{\kappa^{*}}^{1}$ is modular as to $\epsilon^{1} \epsilon^{1}$. This proves the theorem.

The basis stated in the preceding theorem is assumed for the following corollary:

CoRollary. Suppose that $\kappa^{12}$ is by rows of $\overline{\mathfrak{M}}^{2}$. Then $\kappa^{12}$ is modular as to $\epsilon^{1} \epsilon^{2}$ if and only if every vector modular as to $J^{2} \kappa^{12} \kappa^{* 21}$ is modular as to $\epsilon^{1}$.

The transitivity for accordance and finiteness is stated in the following theorem:

\footnotetext{
${ }^{8}$ Moore, G. A., I, p. 22.
} 
Theorem IV. Consider the basis $\mathfrak{A}, \mathfrak{B}, \epsilon$ and let $\epsilon_{0}$ be a positive hermitian matrix. Then

(a) every vector accordant as to $\epsilon_{0}$ is accordant as to $\epsilon$ if and only if $\epsilon_{0}$ is accordant as to $\epsilon \epsilon$;

(b) every vector finite as to $\epsilon_{0}$ is finite as to $\epsilon$ if and only if $\epsilon_{0}$ is of type $F \bar{F}$.

In part (a), if every vector accordant as to $\epsilon_{0}$ is accordant as to $\epsilon$, then $\epsilon_{0}$, being of type $A_{0} \bar{A}_{0}$, is of type $A \bar{A}$. By Theorem (46.5) in G.A., $\epsilon_{0}$ is accordant as to $\epsilon \epsilon$. Conversely every vector $\xi$ accordant as to $\epsilon_{0}$ satisfies the relation $\xi=J_{0} \epsilon_{0} \xi=L_{\sigma} J_{0} \epsilon_{0} \xi_{\sigma}$. Now $J_{0} \epsilon_{0} \xi_{\sigma}$, being a finite (right) linear combination of the columns of $\epsilon_{0}$, is a vector accordant as to $\epsilon$ for every $\sigma$. By Theorem (48.2) in G.A., $\xi$ is accordant as to $\epsilon$. Part (b) is an immediate consequence of the definition of finiteness.

The University of Chicago 